\title{
Advanced Multivariate Analysis Tools Applied to Surface Analysis
}

\author{
James A. (Tony) Ohlhausen*
}

*PO BOX 5800, MS 0886, Sandia National Laboratories, Albuquerque, NM 87185

Surface analysis, the chemical analysis of the topmost atom layers of a material, is a powerful tool for materials analysis. Surface analytical techniques are well suited for the analysis of contamination, corrosion products, and compositional changes due to aging. Typical laboratorybased techniques include X-ray Photoelectron Spectroscopy (XPS) and Time-of-Flight Secondary Ion Mass Spectrometry (ToF-SIMS). Other techniques such as Near Edge X-ray Absorption Fine Structure (NEXAFS) require synchrotron radiation at a dedicated facility. Each of these techniques provides unique elemental and chemical information from the surface of interest.

Modern surface analytical instruments have the ability to acquire images with full spectra at each pixel (spectrum images), and can generate a large amount of data very quickly. Additionally, these techniques can be operated in a mode where spectrum image acquisitions are interleaved with ion sputtering. These types of depth profiles can provide 3D information from the surface at high depth resolutions; however a problem arises when attempting to interpret the data. The magnitude of data, coupled with spatial distortions and instrument artifacts (some unknown) in the technique present a data set that is difficult to comprehensively interpret. Univariate methods are traditionally used to interrogate a data set, but small features in spectral shapes and unknown artifacts can sometimes be overlooked and all correlations between species can be difficult to determine. Multivariate analysis, a statistical approach to these data, appears to be the optimum way to ensure that all data are considered within an analysis.

Multivariate analysis methods have been applied to several surface analytical techniques with great success. Specifically, ToF-SIMS and XPS have benefited from multivariate procedures. Recently, multivariate analysis has been applied to imaging NEXAFS as well. We have found that multivariate analysis provides a comprehensive inventory of the acquired data contained in the full spectrum image. Additionally, the spectra from a multivariate analysis are more easily interpreted and spectral correlations are automatically determined. Examples illustrating the benefits of multivariate analysis, advanced algorithms applied to spatial distortion, and their uses will be presented.

In this presentation, I will show several examples of multivariate analysis applied to surface analysis data. A few of these examples are previewed here. In the first example, XPS was used to determine the identity of a stain on a ceramic button (Figure 1). Stains can be extremely difficult to analyze because their miniscule thickness and concentration. Surface analysis with XPS shows quantitative elemental species separated into 3 distinct areas on this sample, indicating contamination originating from a particular processing step during fabrication. Figure 2 shows the 3D analysis of a semiconductor device using ToF-SIMS. In this example, spatial distortion caused by surface topography and ion-induced roughening are applied to the $3 \mathrm{D}$ volume, rendering a more accurate $3 \mathrm{D}$ picture of the sample surface. Figure 3 shows the multivariate analysis of imaging NEXAFS, revealing surface chemical changes and some instrument artifacts that were not previously seen. 


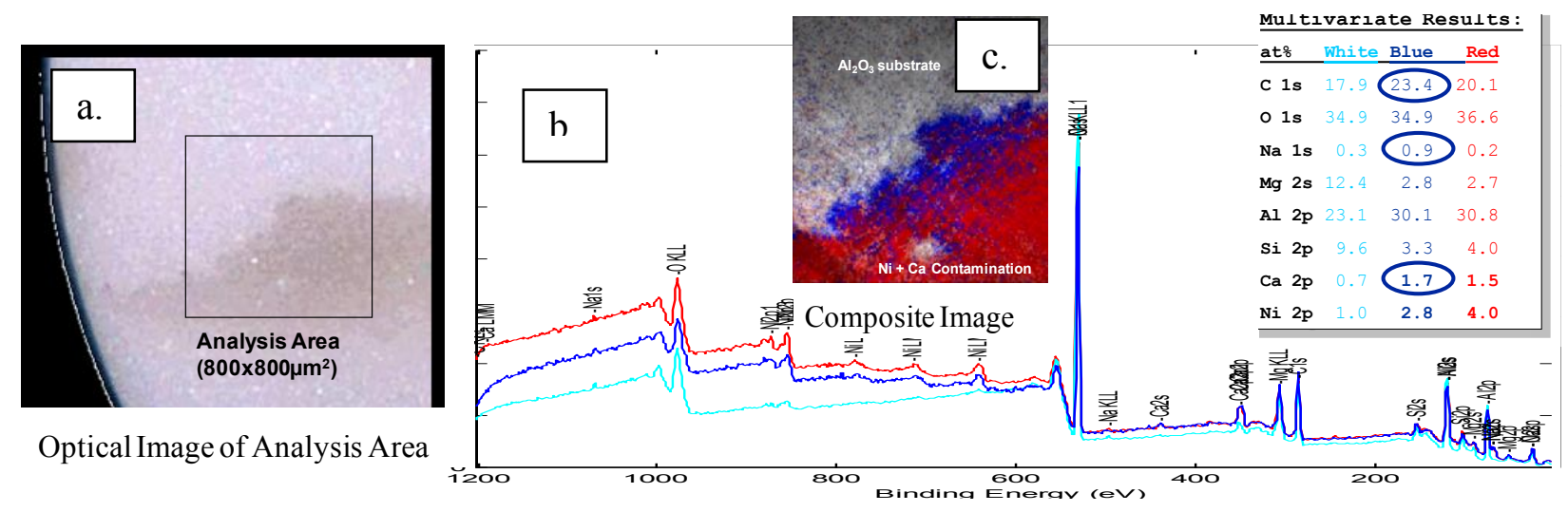

Figure 1: XPS quantitative analysis of a stain on a ceramic disc. The optical photo (a) shows the stain and the XPS analysis area. Multivariate analysis reveals three components, seen by their respective spectra (b) and images (c). Quantitative results from each component reveal the composition of the stain.
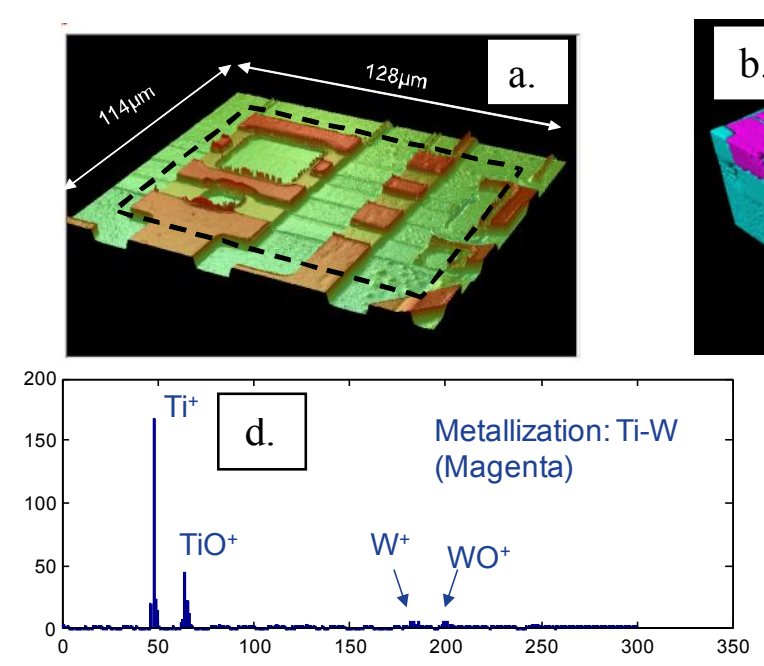
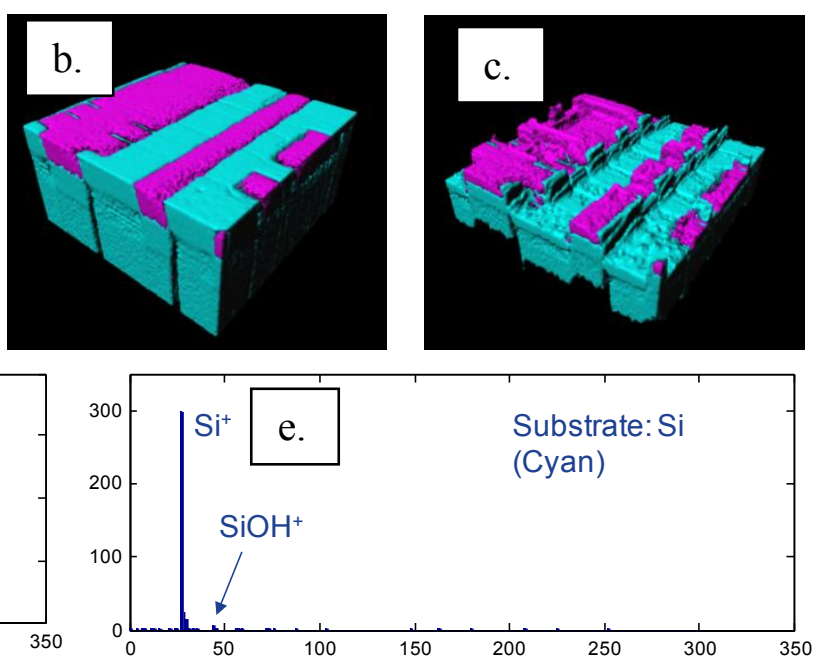

Figure 2: ToF-SIMS 3D multivariate analysis of a semiconductor device. Inset (a) shows an optical profilometry scan of the sample including the analysis area. Uncorrected (b) and corrected (c) 3D volumes are shown. The spectral portions of the multivariate results are presented for the magenta metallization (d) and Si substrate (e).
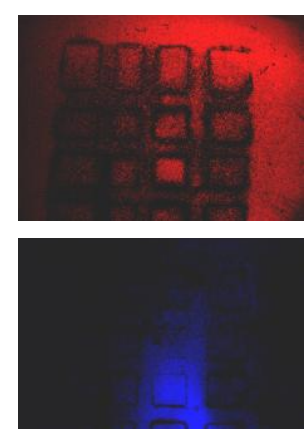
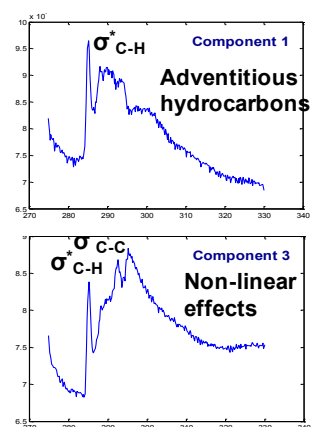
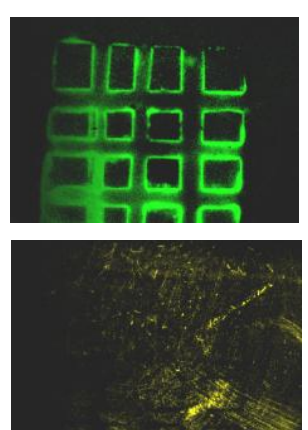
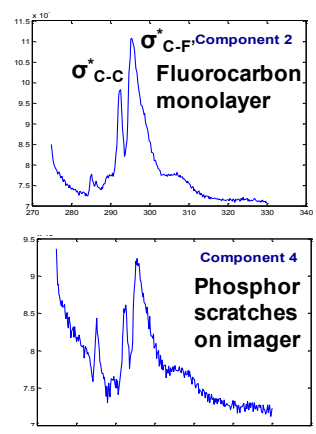

Figure 3: Multivariate results from imaging NEXAFS (18mmx13mm). Components show expected variation across sample, and reveals some instrument artifacts not previously seen. 